\title{
Exceptions in Relational Plurals
}

\author{
Sigrid Beck \\ University of Connecticut
}

\section{Introduction}

This paper gives an account of unusually weak truth conditions in relational plural sentences and reciprocal sentences. The theory of exceptions developed in Brisson (1998) is extended to cumulative and weakly reciprocal interpretations. This strategy reduces the number of semantic readings we need to assume and allows us to capture the influence of context on such data.

In section $1 \mathrm{I}$ introduce the relational plural data that I am interested in and in section 2 the reciprocal data. I point out problems for the analyses currently available. Section 3 introduces my alternative proposal in terms of exceptions.

\section{Weak Truth Conditions for Relational Plurals}

A relational plural is a sentence like (1) (from Scha (1984)) which contains two group denoting expressions (the squares and the circles) and something that expresses a relation between those groups (contain in the example). The question is when we can say that a relation holds between two groups.

(1) The squares contain the circles.

Scha (1984) observes that (1) is true in a situation like s1 given in (2).

$$
\text { s1: }
$$
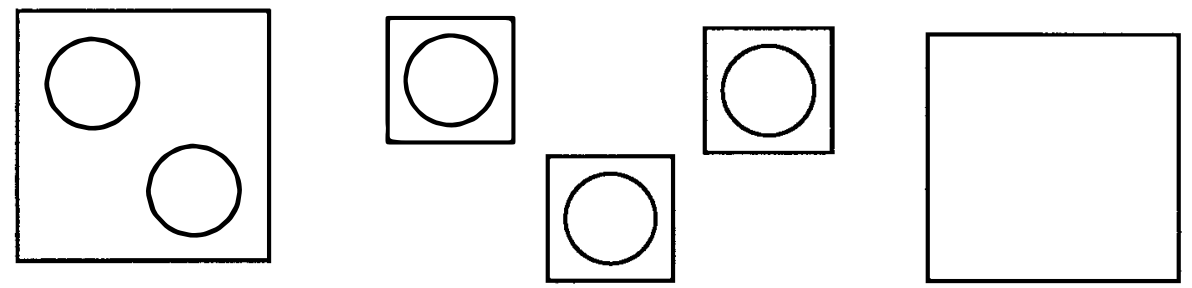

He suggests that the sentence is true just so long as each circle is contained in some square. This amounts to the truth conditions given in (3).

$$
\forall \mathrm{y}[\mathrm{y} \leq \mathrm{C}->\exists \mathrm{x}[\mathrm{x} \leq \mathrm{S} \& \mathrm{x} \text { contains } \mathrm{y}]
$$

(C stands for the group denoted by 'the circles', $\mathrm{S}$ for the denotation of 'the squares' and ' $\leq$ ' is the part-of relation. Assume as a default that the relevant parts of a group are the individual members of that group, i.e. the individual circles and squares. The examples to come will be formalized in an analogous way.)

Scha accounts for this interpretation with the meaning postulate for contain given in (4), which specifies what it means for the 'contain'-relation to hold between two groups. If one were to view this as a general interpretational 
possibility for relational plurals, a relational plural sentence 'ARB' would have, on one reading, the truth conditions given in (5).

$$
\operatorname{contain}(A, B) \text { iff } \forall y[y \leq B->\exists x[x \leq A \& x \text { contains } y]
$$

$$
\text { ARB iff } \forall y[y \leq B->\exists x[x \leq A \& x R y]
$$

So, a group A stands in relation $\mathrm{R}$ to a group $\mathrm{B}$ just in case each member of $\mathrm{B}$ has a member of $A$ standing in relation $R$ to it.

I think that we do not want to view this as a generally available reading of relational plurals because the truth conditions are too weak. This can be seen in two ways. First, let's change the situation and evaluate (1) against situation s2 given in (6).

(6) s2:
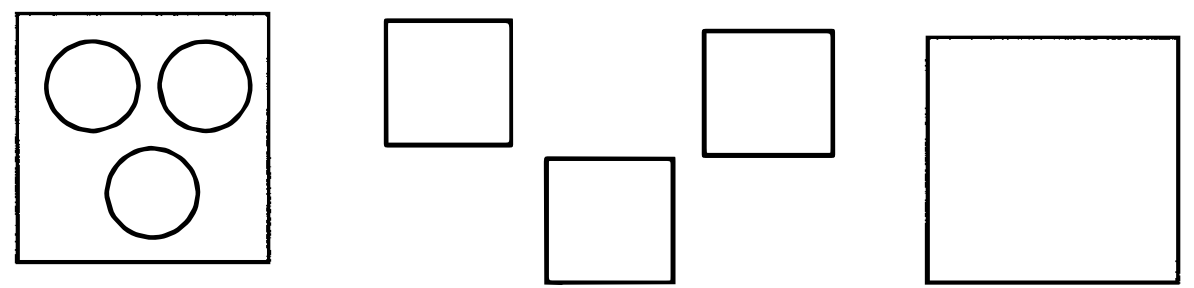

It is at best unclear to me whether (1) is true in s2. If the interpretation were as in (3), I should have no such doubts. The situation is just an extreme case of the truth conditions in (3). Thus, it seems that it is too weak a requirement that only one square needs to be involved.

Secondly, let's change the example slightly, and consider (7).

a. The green jar and the purple jar contain the Earl Grey and the Assam.

b. The three triangles contain the four circles.

Imagine one of the jars is empty; the sentence would intuitively be false. Similarly for (7b). The intuitive truth conditions for (7a) are given in (8). Each jar contains one of the teas and each tea is contained in one of the jars.

$$
\forall \mathrm{x}[\mathrm{x} \leq \mathrm{J} \rightarrow \exists \mathrm{y}[\mathrm{y} \leq \mathrm{T} \& \mathrm{x} \text { contains } \mathrm{y}] \& \forall \mathrm{y}[\mathrm{y} \leq \mathrm{T} \rightarrow \exists \mathrm{x}[\mathrm{x} \leq \mathrm{J} \& \mathrm{x} \text { contains } \mathrm{y}]
$$

This is a cumulative interpretation. Cumulative readings can generally be represented as in (9). Compare (5) to (9): one direction of the cumultive interpretation is missing in the truth conditions suggested by Scha.

(9) cumulation:

$$
\text { ARB iff } \forall x[x \leq A->y[y \leq B \& x R y] \& \forall y[y \leq B->\exists x[x \leq A \& x R y]
$$

The observations regarding (6) and (7) versus (1) suggest a different perspective on why (1) may be judged true in s1. Perhaps we are willing, under appropriate circumstances, to allow an exception to a cumulative interpretation. Then, the second direction of (9) would not really be missing. It's just that we tolerate an exceptional empty square. The conditions under which this is possible will have to 
be clarified. I specify in (10) what would count as an exception to a cumulative reading.

$$
\begin{aligned}
& \text { a. } \exists x[x \leq A \& \sim \exists y[y \leq B \& x R y]] \\
& \text { b. } \exists y[y \leq B \& \sim \exists x[x \leq A \& x R y]]
\end{aligned}
$$

Exceptions are possible in two ways: there might be a member of group A that does not stand in relation $\mathrm{R}$ to any member of $\mathrm{B}$ (a non-containing square); or there might be a member of $B$ that is not Red by any member of $A$ (that would be an uncontained circle in the example). We have seen an instance of a (10a) type exception. I will come back to (10) and to (1) vs. (6) and (7) when we have a theory of exceptions.

\section{Weak Truth Conditions for Reciprocals}

First, let's look at the second set of data I want to discuss. These are reciprocal sentences such as (11) (from Dalrypmle et al. (1998)).

(11) The pirates stared at each other.

Dalrymple et al. (1998) observe that the sentence is judged true in a situation like s3 depicted in (12). The arrow symbolizes the relation 'stare at'.

s3:

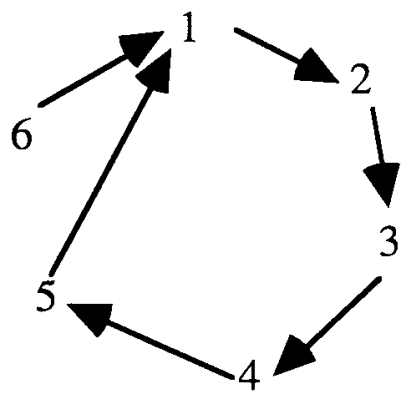

They propose that the sentence is true just so long as each pirate stared at one other pirate, i.e. they propose the truth conditions in (13).

$$
\forall \mathrm{x}[\mathrm{x} \leq \mathrm{P}->\exists \mathrm{y}[\mathrm{y} \leq \mathrm{P} \& \mathrm{x} \neq \mathrm{y} \& \mathrm{x} \text { stared at } \mathrm{y}]]
$$

Quite generally they suggest that reciprocal sentences can have the interpretation in (14), one-way weak reciprocity. A group A stands in relation $\mathrm{R}$ to each other iff each member of $A$ stands in relation $R$ to at least one other member.

One way Weak Reciprocity (OWR):

A $R$ each other iff $\forall x[x \leq A-\exists y[y \leq A \& x \neq y \& x R y]]$

Notice that this reciprocal interpretation is just like Scha's interpretation of the relational plural in terms of quantification over group members. I have similar complaints about OWR as I did about Scha's schema: the truth conditions we 
assign seem, in general, too weak. In (15) I once more change the situation that we evaluate (11) against to the extreme case that would make (13) true: the pirates all stare at the same pirate.

s4:

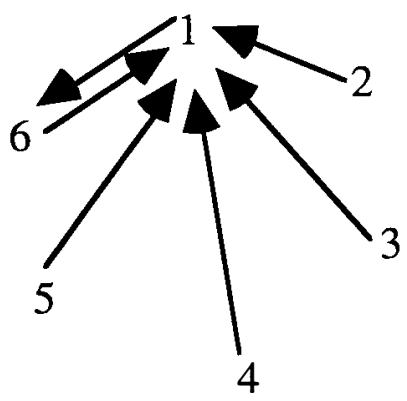

Just as in the case of the squares, it is at best unclear to me whether or not the sentence is true in this situation. And again, according to the truth conditions proposed for the sentence I should not have such doubts, the sentence should be judged true. It seems to be too weak to require that only one member of the pirate group needs to be stared at.

Problems also arise if we change the example. Consider (16a).

(16) a. These three people want to marry each other.

b.

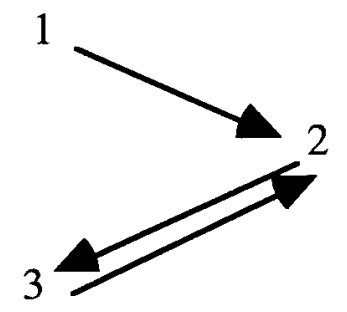

c.

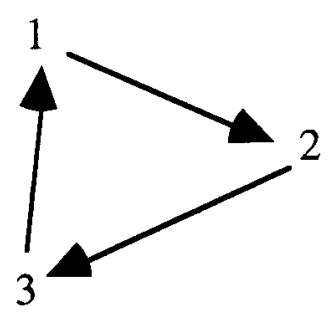

I judge the sentence false in situation (16b) because nobody wants to marry person 1. The sentence would be true in the situation depicted in (16c). The appropriate truth conditions seem to be the ones given in (17): each of the three people wants to marry another one of the three, and for each of the three people there is another one among them who wants to marry that person.

$$
\begin{aligned}
& \forall x[x \leq 3 P->\exists y[y \leq 3 P \& x \neq y \& x \text { wants to marry } y] \& \\
& \forall y[y \leq 3 P->\exists x[x \leq 3 P \& x \neq y \& x \text { wants to marry } y]
\end{aligned}
$$


These truth conditions amount to a weakly reciprocal interpretation. The general case is given in (18) (cf. Langendoen (1978)): we can say that A R each other iff each member of $A$ stands in relation $R$ to one other member of $A$, and also there is another member of $\mathrm{A}$ that stands in relation $\mathrm{R}$ to that individual.

$$
\begin{aligned}
& \text { Weak Reciprocity: } \\
& \text { A R each other iff } \\
& \forall x[x \leq A \rightarrow \exists y[y \leq A \& x \neq y \& x R y]] \& \forall y[y \leq A \rightarrow \exists x[x \leq A \& x \neq y \& x R y]]
\end{aligned}
$$

Comparing WR to OWR, the second direction of WR is missing in OWR. But once more, our observations in this section suggest that perhaps the second direction is not really missing. Perhaps what happens in (11) is rather that, for some reason, we are willing to tolerate an exception to an interpretation that is basically weakly reciprocal (an unstared-at pirate). I specify in (19) what it means to be an exception to a weakly reciprocal interpretation.

$$
\begin{aligned}
& \text { a. } \quad \exists x[x \leq A \& \sim \exists y[y \leq A \& x \neq y \& x R y]] \\
& \text { b. } \quad \exists y[y \leq A \& \sim \exists x[x \leq A \& x \neq y \& x R y]]
\end{aligned}
$$

Either, you are a member of $\mathrm{A}$ that does not stand in relation $\mathrm{R}$ to any other member of A (a non-staring pirate); or you are a member of A that no other member of $\mathrm{A}$ stands in relation $R$ to (an unstared-at pirate). We have seen in s3 in (12) an example of a (19b) type exception. Actually the reverse situation and a type (19b) exception seems to be equally acceptable for this example (reverse the arrow going from pirate 6 to pirate 1 - the situation would still make (11) true). Once more, we will need to know more about exceptions in order to understand the difference between (11) and (16).

\section{An Alternative}

It is now time to make precise this talk about exceptions. Brisson (1998) develops a theory of how certain individuals can be disregarded for the evaluation of a plural predication, and when this is possible. I introduce her theory in section 3.1., and apply it to relational plurals and reciprocals in sections 3.2 and 3.3. respectively. Section 3.4. is a speculation on how many exceptions we might be willing to tolerate.

\subsection{Brisson (1998) and Ill-fitting Covers}

The basic problem Brisson is interested in is illustrated by (20a) vs. (20b).

(20) a. The children built a raft.

b. The children all built a raft.

(20a) has a collective and a distributive reading. Both are compatible with a child that is not involved in raft building. (20b) also has a collective and a distributive reading. However, both are incompatible with an uninvolved child. (20b) does not tolerate exceptions. I concentrate in my discussion on the distributive reading, for convenience. The standard representation of that reading is given in (21). 


$$
\forall \mathrm{x}[\mathrm{x} \leq \mathrm{C} \rightarrow \mathrm{x} \text { built a raft }]
$$

Let's assume as before that the default parts of the children-group are the individual children. (21) says that each of the children built a raft; the standard representation thus does not allow for uninvolved children. Why then are we prepared to tolerate them in (20a) but not in (20b)? To answer this question, Brisson builds on Schwarzschild's (1996) theory of distributivity. The crucial ingredient of this revised theory of distribution is that distribution contains a pragmatic element, which determines what parts of a given group are relevant to distribution. The original motivation for this pragmatic element comes from data like (22a) vs. (22b) (from Schwarzschild (1996)).

(22) a. The cows and the pigs filled the barn to capacity.

b. The young animals and the old animals filled the barn to capacity.

(22a) has a distributive reading that amounts to (23a). (22b) has a distributive reading that amounts to (23b).

(23) a. The cows filled the barn to capacity and the pigs filled the barn to capacity.

b. The young animals filled the barn to capacity and the old animals filled the barn to capacity.

Imagine now that the cows and the pigs are all the animals that there are in the context, and that's just the young animals and the old animals - i.e. assume (24).

$$
\begin{aligned}
{[[\text { the cows and the pigs }]] } & =[[\text { the young animals and the old animals }]] \\
& =[[\text { the animals }]]
\end{aligned}
$$

Then, under standard assumptions the distributive reading of both (22a) and (22b) should be as in (25). But then the interpretational difference between the two is quite mysterious.

$$
\forall \mathrm{x} \text { [ } \mathrm{x} \leq \text { [[the animals]] } \rightarrow \mathrm{x} \text { filled the barn to capacity] }
$$

The intuitive difference lies in what subgroups distribution is down to. It seems that those subgroups of the animals are relevant that are explicitly mentioned. Schwarzschild suggests that the context provides a division into subgroups formally: a cover. The notion of cover is defined as in (26).

$\mathrm{C}$ is a cover of $\mathrm{P}$ iff

$C$ is a set of subsets of $P$

Every member of $\mathrm{P}$ belongs to some set in $\mathrm{C}$

\{\} is not in $\mathrm{C}$

The proposal is that the context provides a cover of the universe of discourse. Distribution is sensitive to that cover. A better representation of the distributive reading of (22) is therefore (27) with a free variable Cov ranging over covers, which will be assigned a value according to context. The cover chosen contains the salient subgroups. 
$\forall \mathrm{x}$ [ $\mathrm{x} \leq[$ [the animals] $] \& \operatorname{Cov}(\mathrm{x})->\mathrm{x}$ filled the barn to capacity]

(27) says that each salient subgroup of the animals filled the barn to capacity. What exactly this amounts to depends on what Cov is. Let's assume that things that are explicitly mentioned are salient. The cover salient for (22a) would then be (28a). With that value for Cov, (27) says that the cows filled the barn to capacity and so did the pigs. That is the desired interpretation (23a). In (22b) on the other hand, mentioning the animals in terms of the young animals and the old animals makes salient the cover in (28b). With that choice of cover, (27) amounts to (23b), the interpretation we intuitively get for $(22 b)$.

a. $\operatorname{Cov}[$ the animals $]=\{[$ the cows $],[[$ the pigs $]]\}$

b. $\operatorname{Cov}[$ the animals $]=\{[[$ the young animals $]]$, [[the old animals $]]\}$

Equipped with this more sophisticated theory of distribution, let's go back to Brisson's example and the exceptions. A more adequate representation of $(20 a, b)$ repeated as $(29 a, b)$ is $(29 c)$ with the added restriction that the parts of the children group be members of the cover. (29c) says that those subparts of the children that are members of the cover built a raft.

$$
\begin{aligned}
& \text { a. The children built a raft. } \\
& \text { b. The children all built a raft. } \\
& \text { c. } \forall x[x \leq C \& \operatorname{Cov}(x)->x \text { built a raft }]
\end{aligned}
$$

How does the cover help us with the problem of (20a) vs. (20b)? Since this does not seem to be a problem with subgroups, let's ignore subgroups and assume once more that distribution is to individual children (this is what Schwarzschild suggests as the default if we have no reason to believe that any interesting subgroups are relevant; we have assumed this so far and will continue to do so). We made an important assumption in our discussion of the examples above, namely that the cover exhausts the groups distributed over; or in other words, that the cover of the universe of discourse is also a cover of the group we are looking at. In our particular example the covers were chosen in such a way that all animals got cought in the distribution by being part of an animal group that is a cell in the cover. Let's make this assumption explicit. For any plurality $\mathrm{Y}$, we want to talk about the covered part of $\mathrm{Y}$ as defined in (30).

$$
\operatorname{Cov}[\mathrm{Y}]:=\{\mathrm{X}: \mathrm{X} \in \operatorname{Cov} \& \mathrm{X} \leq \mathrm{Y}\}
$$

The covered part of $\mathrm{Y}$ is the collection of all those cells in the cover that consist exclusively of members of Y. Looking at the representation of the distributive reading in (29c), the covered part of $C$ is that part of the children group that is quantified over. Imagine that the cover has the property in (31a): if we form the union of all cells in the cover that consist of children only, we get back the original group. Then, each child will be affected by the universal quantification in (29c), and all children without exception will be involved in raft building. This is what Brisson calls a good fit: the cover is also a cover of the plurality under consideration. Imagine on the other hand that the covered part of the children is actually smaller than the original group. Whatever is not included in the covered part of $\mathrm{C}$ will not be affected by the universal quantification in (29c). Those children will be exceptions permitted by (29c): children not involved in raft building. This is what Brisson calls an ill-fitting cover. 

a. $\quad \cup \operatorname{Cov}[\mathrm{C}]=\mathrm{C}$
no exceptions
b. $\quad \cup \operatorname{Cov}[\mathrm{C}]<\mathrm{C}$
exceptions

The semantic contribution of 'all' is that it forces the cover to be a good fit. This accounts for the contrast in (20).

Brisson identifies several factors that affect tolerance of exceptions. One is group size: exceptions are easier to accept in large groups than in small. Another one is information about the structure of the group. It seems that the more you're told about a group, the less you're willing to disregard parts of it. In (32a) for example, in addition to the group being small, the group is specified by naming individual members. It is hard to accept the sentence as true if one of Joe and Stacey is not involved in raft building. Only a special context can achieve that (for example, a team credit kind of scenario); I refer the interested reader to Brisson (1998) for discussion.
a. Joe and Stacey built a raft.
b. The three children built a raft.

I suggest that counting group members as in (32b) has an effect similar to naming group members: it makes no sense to introduce the raft builders as 'the three children' if I then want to disregard part of that group in the evaluation of the plural predication.

To sum up, we now have a partially pragmatic theory of distribution. The context contributes a cover of the universe of discourse, which serves two functions: it accounts for subgroup effects, and it provides a mechanism by which we can accept exceptions to a plural predication.

\subsection{Covers in Relational Plurals}

I propose to carry over this theory of exceptions to relational plurals. In particular, I suggest that cumulative readings are sensitive to covers as well. Since covers are the mechanism by which we get exceptions, we expect them to be tolerated in cumulative interpretations as well as distributive interpretations.

The original motivation for covers are subgroup effects, and subgroup effects showing up in cumulative readings provide independent evidence that cumulation is sensitive to covers. Consider (33).

(33) The female cows and the female goats outnumber the male cows and the male goats.

A salient interpretation for this sentence is one in which it would be true, for example, in a situation in which the female cows outnumber the male cows and the female goats outnumber the male goats. This is a cumulative interpretation relative to subgroups. A representation is given in (34) and the probable cover of the animals in (35). 
$\forall \mathrm{x}[\mathrm{x} \leq[$ [the female cows and the female goats] $] \& \operatorname{Cov}(\mathrm{x})->$

$\exists \mathrm{y}[\mathrm{y} \leq[[$ the male cows and the male goats $]] \& \operatorname{Cov}(\mathrm{y}) \&$ outnumber( $\mathrm{x}, \mathrm{y})]]$

$\& \forall \mathrm{y}[\mathrm{y} \leq[$ [the male cows and the male goats] $] \& \operatorname{Cov}(\mathrm{y})->$

$\exists x[x \leq[[$ the female cows and the female goats $]] \& \operatorname{Cov}(\mathrm{x}) \&$ outnumber $(\mathrm{x}, \mathrm{y})]]$

(35) $\operatorname{Cov}[$ the animals $]=\{F C, F G, M C, M G\}$

That is, each salient subgroup of the female animals outnumbers a salient subgroup of the male animals, and each salient subgroup of the male animals is outnumbered by a salient subgroup of the female animals.

This means that we should revise the semantics of cumulation as in (9'). (9') is just like (9) except that there is an extra condition that the subparts of our two groups be elements of the cover. In other words, the subgroups introduced in the quantification are required to be salient subgroups.

(9') cumulation:

$\mathrm{ARB}$ iff $\quad \forall \mathrm{x}[\mathrm{x} \leq \mathrm{A} \& \operatorname{Cov}(\mathrm{x}) \rightarrow \exists \mathrm{y}[\mathrm{y} \leq \mathrm{B} \& \operatorname{Cov}(\mathrm{y}) \& \mathrm{xRy}] \&$

$\forall \mathrm{y}[\mathrm{y} \leq \mathrm{B} \& \operatorname{Cov}(\mathrm{y})->\exists \mathrm{x}[\mathrm{x} \leq \mathrm{A} \& \operatorname{Cov}(\mathrm{x}) \& \mathrm{xRy}]$

Equipped with this revised theory of cumulation, let's reconsider the Scha sentence repeated below.

(36) The squares contain the circles.

The cumulative reading of this sentence is given in (37), which includes reference to the cover.

$$
\begin{aligned}
& \forall \mathrm{x}[\mathrm{x} \leq S \& \operatorname{Cov}(\mathrm{x})->\exists \mathrm{y}[\mathrm{y} \leq C \& \operatorname{Cov}(\mathrm{y}) \& \mathrm{x} \text { contains } \mathrm{y}] \& \\
& \forall \mathrm{y}[\mathrm{y} \leq C \& \operatorname{Cov}(\mathrm{y})->\exists \mathrm{x}[\mathrm{x} \leq S \& \operatorname{Cov}(\mathrm{x}) \& \mathrm{x} \text { contains } \mathrm{y}]
\end{aligned}
$$

Imagine that the cover has the properties in (38).

$$
\cup \operatorname{Cov}[S]<S \quad \cup \operatorname{Cov}[\mathrm{C}]=\mathrm{C}
$$

So, while the cover is also a cover of the circles, it is ill-fitting with respect to the squares. This means that there is a square that is not affected by the quantification in (37). In other words, with a cover choice as described in (38), (37) will be compatible with a square that does not contain any of the circles. Such a square will be a permissible exception to a cumulative reading.

The data in (7) (repeated below as (39)) are examples in which exceptions were not acceptable. These data can now be identified as naming and counting effects on a par with (32).

(39) a. The green jar and the purple jar contain the Earl Grey and the Assam. b. The three triangles contain the four circles.

And finally, the fact that (36) is hard to accept as true in situation s2 in (6) makes some intuitive sense now: we would have to allow quite a lot of exceptions to our basic cumulative interpretation. Plausibly this is dispreferred and would require a special context to be acceptable, and we have not provided such a context. 
This theory of exceptions in cumulative readings makes some further predictions. Most importantly perhaps, we expect exceptions to be possible in both ways indicated in (10); that is, there can be a non-covered part to both the subject and the object group in a relational plural. This is implausible for the example we have been looking at. In general however this prediction is borne out. A pertinent example is given in (40a), which is once more taken from Scha (1984).

(40) a. The sides of rectangle 1 cross the sides of rectangle 2.

b. $\forall \mathrm{x}[\mathrm{x} \leq \mathrm{SR} 1 \& \operatorname{Cov}(\mathrm{x})->\exists \mathrm{y}[\mathrm{y} \leq \mathrm{SR} 2 \& \operatorname{Cov}(\mathrm{y}) \& \mathrm{x}$ crosses $\mathrm{y}] \&$

$\forall \mathrm{y}[\mathrm{y} \leq \mathrm{SR} 2 \& \operatorname{Cov}(\mathrm{y}) \rightarrow \exists \mathrm{x}[\mathrm{x} \leq \mathrm{SR} 1 \& \operatorname{Cov}(\mathrm{x}) \& \mathrm{x}$ crosses $\mathrm{y}]$

Note first that a cumulative reading (in which each side of $\mathrm{R} 1$ crosses at least one side of R2 and vice versa) is the strongest possible interpretation of this sentence; in particular, a stronger reading like a doubly distributive interpretation (in which each side of R1 crosses every side of R2) is factually impossible. Let's therefore assume the cumulative interpretation given in (40b) as the basic reading of this sentence. Now, Scha observes that (40a) is judged true in the situation given in (41).

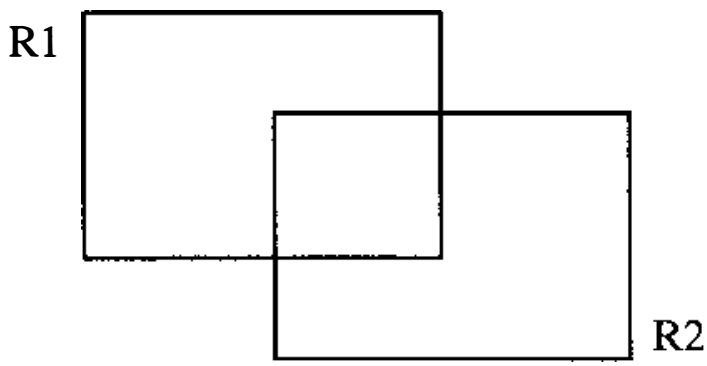

This is what we would expect, given our claim that the cumulative reading of the sentence looks as in (40b). The cover variables contained in (40b) will allow for exceptions should the context support it. (42) shows what would count as an exception to (40): a side of R1 that does not cross any side of R2, or a side of R2 not crossed by any side of $\mathrm{R} 1$.

$$
\begin{aligned}
& \text { a. } \quad \exists x[x \leq S R 1 \& \sim \exists y[y \leq S R 2 \& x \text { crosses } y]] \\
& \text { b. } \exists y[y \leq S R 2 \& \sim \exists x[x \leq S R 1 \& x \text { crosses } y]]
\end{aligned}
$$

(40) permits both kinds of exceptions. I should note at this point that Scha's analysis of this example is not in terms of exceptions of course. He suggests the truth conditions given in (43), which are derived from the lexical meaning of cross as it holds between two pluralities.

$$
\exists x[x \leq S R 1 \& \exists y[y \leq S R 2 \& x \text { crosses } y]]
$$

My criticism of (5) as a general pattern for the truth conditions of a relational plural generalizes to (43).

I would like to include the non-geometric example in (44a) in the discussion, which makes the same point but which I find a bit easier to judge. 
a. The forwards are faster than the defenders.

b. $\forall \mathrm{x}[\mathrm{x} \leq \mathrm{F} \& \operatorname{Cov}(\mathrm{x}) \rightarrow \exists \mathrm{y}[\mathrm{y} \leq \mathrm{D} \& \operatorname{Cov}(\mathrm{y}) \& \mathrm{x}$ is faster than $\mathrm{y}] \&$ $\forall \mathrm{y}[\mathrm{y} \leq \mathrm{D} \& \operatorname{Cov}(\mathrm{y})->\exists \mathrm{x}[\mathrm{x} \leq \mathrm{F} \& \operatorname{Cov}(\mathrm{x}) \& \mathrm{x}$ is faster than $\mathrm{y}]$

Since we are, once more, interested in exceptions to a cumulative reading (and not for example to a stronger distributive reading), let's first make sure that we envision a cumulative interpretation for the example. The background $I$ have in mind is soccer, although depending on the reader's cultural background and athletic preferences other team sports might work as well. The point is that among the several forwards and several defenders, most are unlikely to come up against each other. The sentence is very plausibly taken to make a weaker claim than a doubly distributive reading (where every forward would have to be faster than every defender). Let's focus on the cumulative reading then, which according to our claims should look as in (44b). The cover variables contained in (44b) will allow for exceptions should the context support it. (45) shows what would count as an exception to (44): a forward who is not faster than any defender, and a defender that is not slower than any forward. A (45a) type exception would be a slow center forward who we know is not expected to run, just score when the ball gets to her/him. A (45b) type exception would be a fast sweeper, who we know is not the direct opponent of any forward, but will take care of critical situations that arise when another defender is passed by her/his opponent. Both are possible exceptions.

a. $\quad \exists x[x \leq F \& \sim \exists y[y \leq D$ \& $x$ is faster than $y]]$

b. $\exists y[y \leq D \& \sim \exists x[x \leq F \& x$ is faster than $y]]$

Interestingly, Brisson's analysis of all combined with this theory of cumulation leads us to expect that all is compatible with a cumulative interpretation. This is correct - an example is given in (46a). (46a) is chosen to make exceptions to the cumulative interpretation very implausible. Actually our predictions are a bit more specific: 'all' enforces that the cover be a good fit for the NP that 'all' is associated with. Thus we expect that (46b) is compatible with the fast sweeper but not with the slow center forward, and the reverse for (46c). Again, the predictions are borne out.

a. The three forwards are all faster than the three defenders.

b. The forwards are all faster than the defenders.

c. The forwards are faster than all the defenders.

In (47) below I provide data with the Scha example that are analogous to the data in (46). (47a), while permitting a cumulative interpretation, is intolerant to exceptions, as predicted. (47b) excludes an uncrossed side of $\mathrm{R} 1$, but might still permit an uncrossed side of R2, and the reverse for (47c). Sample situations for (47a-c) are given in (48a-c).

(47) a. The four sides of R1 cross all four sides of R2.

b. The sides of $\mathrm{R} 1$ all cross the sides of R2.

c. The sides of R1 cross all the sides of R2. 
(48)

a.

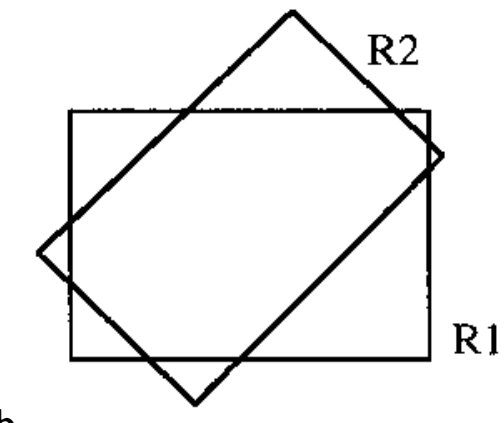

b.

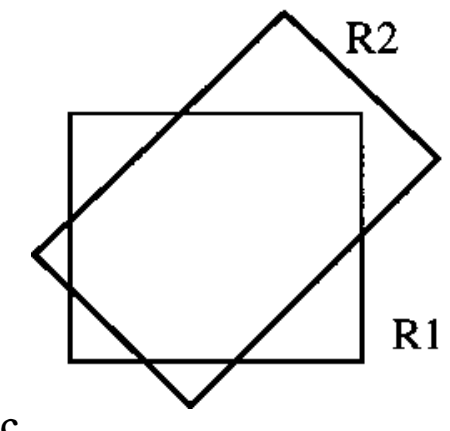

c.

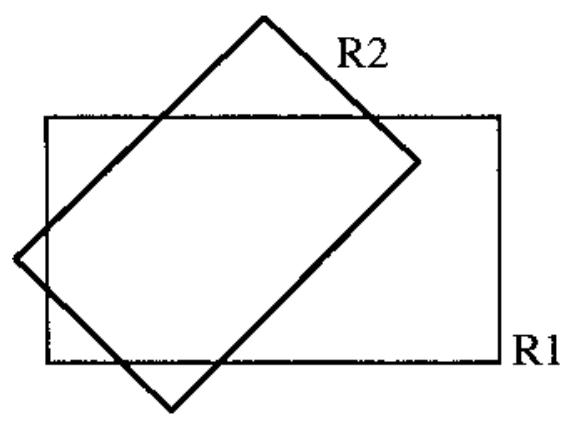

\subsection{WR and Ill-fitting Covers}

In this section I extend the proposal about the role of exceptions to the reciprocal data. This essentially means that weakly reciprocal interpretations must be sensitive to covers, too. (49) below provides independent motiviation for this in terms of subgroup effects in weak reciprocity.

(49) The syntacticians of the two departments and the semanticists of the two departments meet with each other.

Let's concentrate on group meetings. The sentence makes salient four subgroups of the linguists, the ones in (51). It would then be made true by various scenarios of meetings between those groups, for example the syntacticians of department 1 meeting with the syntacticians of department 2 , and the same for the semanticists. The weakly reciprocal interpretation for subgroups that is a salient interpretation for this sentence is given in (50) (I use L 'the linguists' to stand for the referent of 'the syntacticians of the two departments and the semanticists of the two departments'). 
$\forall \mathrm{x}[\mathrm{x} \leq \mathrm{L} \& \operatorname{Cov}(\mathrm{x})->\exists \mathrm{y}[\mathrm{y} \leq \mathrm{L} \& \operatorname{Cov}(\mathrm{y}) \& \mathrm{x} \neq \mathrm{y} \& \mathrm{x}$ meet with $\mathrm{y}]] \&$ $\forall \mathrm{y}[\mathrm{y} \leq \mathrm{L} \& \operatorname{Cov}(\mathrm{y})->\exists \mathrm{x}[\mathrm{x} \leq \mathrm{L} \& \operatorname{Cov}(\mathrm{x}) \& \mathrm{x} \neq \mathrm{y} \& \mathrm{x}$ meet with $\mathrm{y}]]$

(51) $\operatorname{Cov}[$ the linguists $]=\{$ SemD1, SemD2, SynD1, SynD2 $\}$

Thus the weakly reciprocal reading (50) says that each salient subgroup of the linguists meets with some other salient subgroup of the linguists. This means that we ought to revise the semantics of weak reciprocity as shown in (18'). (18') is just like (18) except that the subgroups of the group A are required to be elements of the cover.

(18') Weak Reciprocity:

A $R$ each other iff

$$
\begin{aligned}
& \forall x[x \leq A \& \operatorname{Cov}(x)->\exists y[y \leq A \& \operatorname{Cov}(y) \& x \neq y \& x R y]] \& \\
& \forall y[y \leq A \& \operatorname{Cov}(y) \rightarrow \exists x[x \leq A \& \operatorname{Cov}(x) \& x \neq y \& x R y]]
\end{aligned}
$$

Let's reconsider the pirate example repeated below in the light of these revisions.

The pirates stared at each other.

According to what we just said, the correct representation of the weakly reciprocal reading of $(52)$ is (53) with the cover variables.

$$
\begin{aligned}
& \forall \mathrm{x}[\mathrm{x} \leq \mathrm{P} \& \operatorname{Cov}(\mathrm{x})->\exists \mathrm{y}[\mathrm{y} \leq \mathrm{P} \& \operatorname{Cov}(\mathrm{y}) \& \mathrm{x} \neq \mathrm{y} \& \mathrm{x} \text { stared at } \mathrm{y}]] \& \\
& \forall \mathrm{y}[\mathrm{y} \leq \mathrm{P} \& \operatorname{Cov}(\mathrm{y})->\exists \mathrm{x}[\mathrm{x} \leq \mathrm{P} \& \operatorname{Cov}(\mathrm{x}) \& \mathrm{x} \neq \mathrm{y} \& \mathrm{x} \text { stared at } \mathrm{y}]]
\end{aligned}
$$

Imagine that the cover has the property in (54) - e.g. it does not contain pirate 6. That pirate will not be affected by the quantification over group members in (53), i.e. will be an exception permitted by (53). In other words, pirate 6 is not required to be stared at by any other pirate. The sentence can thus be true in situation $\mathrm{s} 3$ in (12).

$$
\cup \operatorname{Cov}[\mathrm{P}]<\mathrm{P}
$$

Actually, if pirate 6 is not covered, then he will be disregarded not only as a staredat individual but also as a starer. The two ways in which one can be an exception to a weakly reciprocal interpretation are not distinguished by the story as we are telling it here. The sentence should be equally acceptable in a situation like s5 in (55) with a completely uninvolved pirate. This seems fine. 
s5:

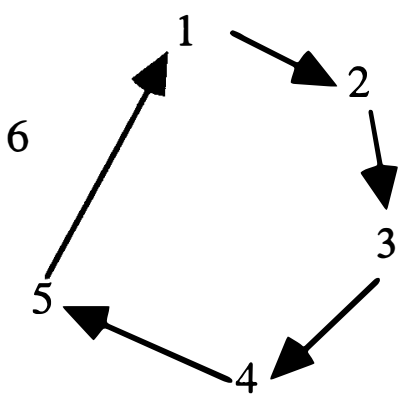

We expect the same naming and counting effects to show up in reciprocal data as in relational plurals. Example (16) repeated in (56a) is just such a case. And we expect the presence of 'all' to preclude exceptions: (56b) in contrast to (52) should be false in $\mathrm{s} 5$, and it is.

(56) a. These three people want to marry each other.

b. The pirates all stared at each other.

Finally, looking back to situation s4 in (15), this seems to be another case in which too many pirates would count as exceptions, and again covers that are that ill-fitting seem dispreferred.

\subsection{The Fit of the Cover}

We saw that the cover is a cover of the universe of discourse, not necessarily a cover of the pluralities affected by plural predication. However, it seems that we still want to impose requirements on the cover as 'covering' those pluralities. At least, we will want to say (57):

$$
\cup \operatorname{Cov}[\mathrm{Y}] \cap \mathrm{Y} \neq\{\}
$$

If no part of a plurality $\mathrm{Y}$ is a member of the cover, quantification will be completely vacuous. That seems illegal, so (57) is certainly a constraint that the cover has to obey. The data discussed above indicate that we will probably want to say a bit more though. It seems that a 'sufficent' part of the plurality we are looking at needs to be covered. This is not a new intuition. Consider (58a), an example from Roberts (1987). (58b) is the description of the situation she wants to evaluate (58a) against: the sentence is true in that situation. (59) is the representation of the intuitive interpretation she suggests for the example (Roberts credits unpublished work by Emmon Bach for this suggestion).

(58) a. The leaves touched each other.

b. The leaves are all on one tree. Most of them touch one or more other leaves.

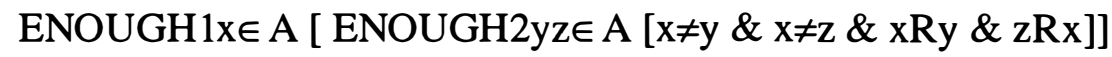

I assume that the vague quantifier ENOUGH has the interpretation in (60). 
$[[$ ENOUGHx $\Phi]] \mathrm{g}=1$ iff $\operatorname{card}(\{\mathrm{a}:[[\Phi]] \mathrm{g}[\mathrm{a} / \mathrm{x}]=1\})>\mathrm{n}$

(where $\mathrm{n}$ is what counts as sufficient in the context)

I will not discuss the second ENOUGH in (59), but rather stick to the intuition behind the paraphrase in (58b) that sufficiently many leaves touch at least one other leaf. (59) can then be simplified to (61a). Compare (61a) to the weakly reciprocal interpretation represented in (61b).
a. ENOUGHx $\in \mathrm{A}$
[ $\exists y z \in A[x \neq y \& x \neq z \& x R y \& z R x]]$
b. $\forall \mathrm{x} \in \mathrm{A}$
[ $\exists y z \in A[x \neq y \& x \neq z \& x R y \& z R x]]$

It seems that the vague quantifier ENOUGH essentially limits the number of exceptions to (61b) (non-touching leaves) that are permitted.

Regarding the use of a quantifier like ENOUGH, Schwarzschild (1996) argues convincingly that counting group members is not in general a good way to evaluate a reciprocal sentence. Consider his example (62).

(62) The prisoners on the two sides of the room could see each other.

The sentence has a prominent reading in which it is true just in case the prisoners on one side of the room can see the prisoners on the other side of the room and vice versa. Imagine an opaque barrier in the middle of the room. The sentence would be false. However, make one group of prisoners very large and the other very small. The resulting numbers will make a claim like (59) true, but intuitively, the sentence remains false. Schwarzschild concludes that the reciprocal relation see has to hold between the salient subgroups of the reciprocal antecedent, not a certain number of members of that group.

For this and related reasons, I will not consider a theory of reciprocals in terms of ENOUGH. But I suggest that we may be able to recover the intuition behind ENOUGH as a constraint on the cover: the cover must cover a sufficient proportion of the group quantified over in our plural predication. Consider (63) as a constraint on the cover.

$$
\operatorname{card}(\cup \operatorname{Cov}[\mathrm{Y}]): \operatorname{card}(\mathrm{Y}) \geq \mathrm{n}
$$

Suppose that $n=1$. Then (63) says that the covered part of $Y$ must be as large as $Y$ itself, i.e. that the cover is also a cover of Y. No exceptions will be permitted. This seems to be one default value for $\mathrm{n}$. But we have seen that $\mathrm{n}$ need not always be 1 , it may be smaller. I don't think we can fix the value for $n$ once and for all; $n$ will be what counts as enough in a given context and will vary from one context to another. I propose to adopt (63) as a vague constraint on the cover.

\section{Conclusion}

I suggest that relational plurals do not have Scha's weaker interpretation in addition to the standard cumulative one; nor do reciprocals have a one-way weakly reciprocal interpretation in addition to a weakly reciprocal one. Weaker readings than cumulative and weakly reciprocal ones are to be analyzed in terms of exceptions. Exceptions to a cumulative reading and a weakly reciprocal interpretation come about by an independent theory of covers. This theory avoids increasing the number of semantic readings we need to assume for plural sentences, 
and it gives us some insight into why tolerance of exceptions varies with the context. The findings in this paper confirm the parallel between relational plurals and reciprocals and support a theoretical perspective that relates weak reciprocity to cumulation, as argued by Langendoen (1978) and Sternefeld (1998).

\section{Acknowledgements}

My thanks to the audiences at the MIT/UConn/UMass workshop II, CUNY graduate center and SALT X, and in particular to Christine Brisson.

\section{References}

Brisson, Christine (1998). Distributivity, Maximality and Floating Quantfiers. PhD dissertation, Rutgers University.

Dalrymple, M., Kanazawa, M., Kim, Y., Mchombo, S. \& Peters, S. (1998). Reciprocal Expressions and the Concept of Reciprocity. Linguistics and Philosophy 21, 159-210.

Langendoen, D. Terence (1978). The Logic of Reciprocity. Linguistic Inquiry 9, 177-197.

Roberts, Craige (1987). Modal Subordination, Anaphora and Distributivity. PhD dissertation, University of Massachusetts at Amherst.

Scha, Remko (1984). Distributive, Collective and Cumulative Quantification. In J. Groenendijk et al. (eds.): Truth, Interpretation and Information, GRASS 2, Foris, Dordrecht, 131-158.

Schwarzschild, Roger (1996). Pluralities. Kluwer, Dordrecht.

Sternefeld, Wolfgang (1998). Reciprocity and Cumulative Predication. Natural Language Semantics 6.3., 303-337. 